\title{
Prótese valvar aórtica sem suporte: o que aprendemos
}

\author{
Vinícius José da Silva NINA* \& Mark F. O’BRIEN*
}

RBCCV 44205-538

\begin{abstract}
Nina V J S \& O’Brien M F - Prótese valvar aórtica sem suporte: o que aprendemos. Rev Bras Cir Cardiovasc 2001; 16(2): 119-27.
\end{abstract}

RESUMO: Objetivo: Analisar o estado atual e a justificativa para o uso continuado das próteses aórticas sem suporte, dando enfoque aos resultados clínicos com a prótese porcina composta CryoLifeO'Brien ${ }^{\mathrm{TM}}$ (CLOB).

Casuística e Métodos: Entre dezembro de 1992 e fevereiro de 2000, 307 pacientes foram submetidos à troca da valva aórtica por uma prótese CLOB. A idade média dos pacientes era de 73 anos (59-89 anos), sendo $16 \%$ acima de 80 anos. Todos os pacientes foram analisados clínica e ecocardiograficamente no pós-operatório com 6 dias, 6 meses, 12 meses e anualmente.

Resultados: A mortalidade hospitalar foi 1,3\% (4 casos não relacionados à troca valvar). A mortalidade tardia foi $9,2 \%$ (28 pacientes); dos quais somente 2 (endocardite tardia) eram prótese-relacionados. Morbidade incluiu: $2(0,6 \%)$ casos de acidente vascular cerebral perioperatório, 6 (1,9\%) "leaks" perivalvares, $6(1,9 \%)$ endocardites e $1(0,3 \%)$ deterioração estrutural. Reoperação foi necessária em 6 (1,9\%) pacientes: 3 por endocardite, 2 por "leak" perivalvar e 1 por deterioração estrutural. Os ecocardiogramas seriados demonstraram um gradiente médio de $7 \mathrm{mmHg}$, com uma baixa incidência de incompetência trivial pelo doppler, e regressão significativa da hipertrofia ventricular esquerda $(p=0,05)$.

Conclusão: As próteses sem suporte têm apresentado excelentes resultados a curto e médio prazos. Entretanto, vigilância rigorosa é necessária para determinar a durabilidade aos 10-12 anos, período em que as valvas porcinas com suporte começam a demonstrar deterioração estrutural.

DESCRITORES: Prótese das valvas cardíacas. Implante de prótese de valva, métodos.

\section{INTRODUÇÃO}

Os primeiros xenoenxertos utilizados para a substituição da valva aórtica no período de 1945 a 1969 eram sem suporte ${ }^{(1-3)}$ e não preservados com glutaraldeído. Aquelas próteses eram preservadas por diferentes métodos e a maioria trazia uma base muscular no folheto coronariano direito. Naquela época, o domínio da preservação dos tecidos estava somente no início. Consequentemente, os resultados clínicos eram pobres, ocorrendo disfunção valvar precoce, o que motivou a interrupção do uso das próteses sem suporte no final de $1969{ }^{(4)}$. Além da preservação tecidual inadequada, havia dois problemas técnicos relacionados ao implante, o primeiro era assimetria de algumas valvas aórticas bicúspides e raízes da aorta, e o segundo a inclusão na linha de sutura do tecido muscular da base do folheto coronariano direito. Ambos os fatores aumentavam a deformidade dos folhetos, causando o aparecimento precoce de insuficiência valvar progressiva.

\footnotetext{
Trabalho realizado no The Prince Charles Hospital, Brisbane, Austrália.

Recebido para publicação em julho de 2000.

* Do Hospital The Prince Charles Hospital.

Endereço para correspondência: Vinícius Nina. Rua Sebastião Archer, 101. Bairro Olho D’Água. São Luís, MA, Brasil. CEP 65065-480. Tel: (98) 2480320.e-mail:vnina33@hotmail.com
} 
Ainda no final da década de 60, CARPENTIER et al. (5) introduziram a valva aórtica porcina com suporte preservada com glutaraldeído. Os problemas pareciam estar resolvidos. A preservação com glutaraldeído era muito superior a do formaldeído e, por outro lado, a valva com suporte não se destinava somente à substituição das valvas atrioventriculares, mas era também a solução para todas as dificuldades técnicas com o implante da valva sem suporte, especialmente nas raízes aórticas dilatadas e assimétricas. Após duas décadas, as limitações das valvas com suporte começaram a ser documentadas (6-16). Dentre elas: degeneração tecidual, calcificação, ruptura ou perfuração dos folhetos.

Infelizmente, as próteses sem suporte preservadas em glutaraldeído não foram testadas nos anos 70 , consequentemente a oportunidade de analisar a durabilidade destas valvas a longo prazo foi perdida. Por esse motivo, as valvas sem suporte disponíveis atualmente no mercado devem ser consideradas em "teste ou estudo".

Constantes reavaliações têm sido feitas para se indicar o melhor substituto valvar. À luz dos conhecimentos atuais, sabe-se que um homoenxerto com suporte dura menos que um homoenxerto sem suporte, e que o mesmo se aplica aos xenoenxertos com e sem suporte que eram preservados com formaldeído (17). Portanto, teoricamente, parece razoável extrapolar que um xenoenxerto sem suporte seria pelo menos igual ou provavelmente superior em durabilidade que um xenoenxerto com suporte para troca da valva aórtica. Além disso, a melhor hemodinâmica e regressão ventricular esquerda(18) associada à crescente perspectiva de uma maior sobrevida a longo prazo têm colocado em evidência estes dispositivos valvares ${ }^{(19)}$.

O objetivo deste estudo é analisar o estado atual e a justificativa para o uso continuado das próteses aórticas sem suporte dando enfoque aos resultados clínicos com a prótese porcina composta CryoLife-O'Brien ${ }^{\mathrm{TM}}(\mathrm{CLOB})$, desenvolvida nesta Instituição.

\section{Aspectos Gerais e Diferenças entre as Próteses sem Suporte}

Nos últimos dez anos, várias companhias têm produzido e comercializado uma variedade de valvas sem suporte, as quais necessitam de uma avaliação cuidadosa e criteriosa. As publicações recentes apresentam uma excelente descrição a respeito das próteses valvares sem suporte disponíveis no mercado(17-21).

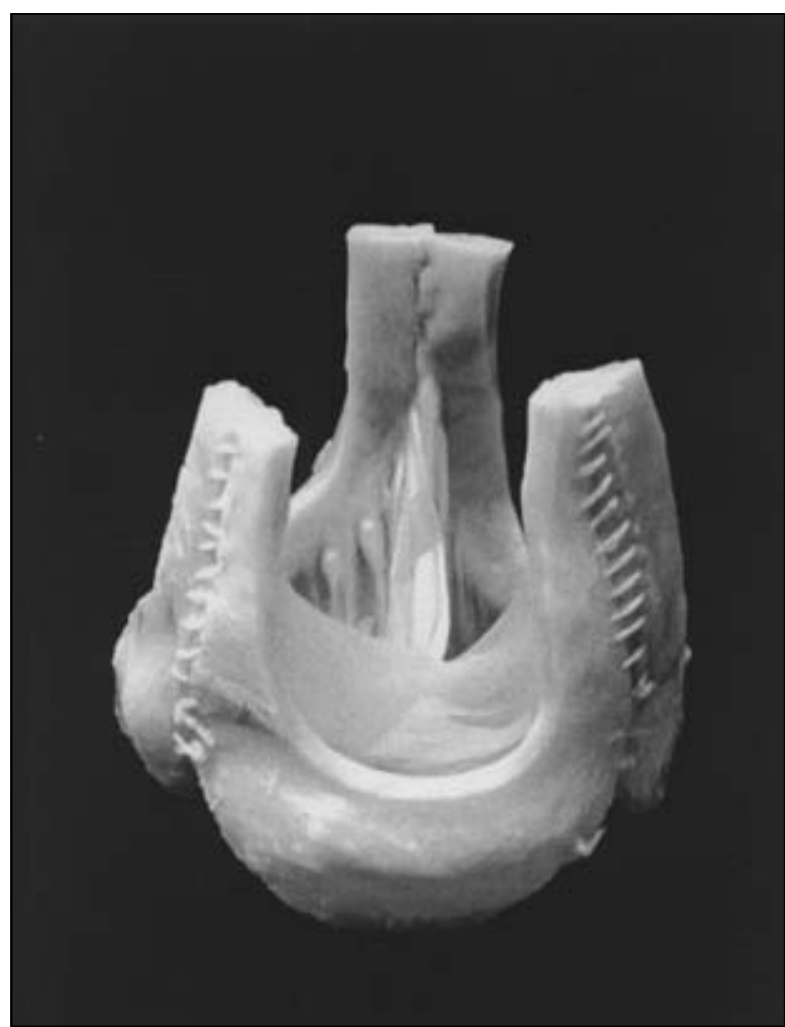

Fig. 1 - Xenoenxerto porcino sem suporte CryoLife O’BrienTM composto de 3 folhetos não coronarianos (reproduzida com permissão do Dr. Mark F. O’Brien).

Ainda não é conhecida a influência das variações do modelo na durabilidade dessas próteses. A maioria das valvas sem suporte têm um revestimento externo de Dacron que pode ser: completo (Baxter / Prima ${ }^{\mathrm{TM}}$ e Toronto SPV ${ }^{\mathrm{TM}}$ ) ou parcial recobrindo apenas a base muscular do folheto coronariano direito (Medtronic Freestyle ${ }^{\mathrm{TM}}$ ). A valva CryoLife-O'Brien ${ }^{\mathrm{TM}}$ por ser composta sem o folheto coronariano direito, não requer suporte de Dacron (Figura 1).

A principal diferença entre os modelos disponíveis não se relaciona apenas ao revestimento externo de Dacron, mas também à quantidade de tecido, seja muscular ou da parede aórtica que é retida na porção proximal e distal do xenoenxerto. $A$ valva CLOB tem pouco tecido aórtico distal, e não tem remanescente tecidual proximal, portanto requer apenas uma linha de sutura contínua para o seu implante; enquanto que as demais próteses, devido à construção anatômica, requerem diferentes técnicas de implante dentre as quais: implante subcoronário, cilindro intra-aórtico ou substituição da raiz da aorta. A utilização de uma destas 3 técnicas pressupõe a confecção de duas linhas de sutura (22). 
Além disso, é possível que o excesso de tecido na parede do xenoenxerto possa contribuir para calcificação e em conseqüência, menor durabilidade. Alguns efeitos parecem ser determinantes da durabilidade tardia:

- Vários métodos de fixação com glutaraldeído têm proporcionado resultados clínicos variáveis. As vantagens clínicas da pressão de fixação zero ainda não estão bem compreendidas. Vários autores relataram que a grande vantagem da pressão de fixação zero reside na capacidade de retenção do colágeno pelos folhetos, embora isto possa ser perdido gradualmente in vivo (23-25).

- Concentrações variáveis de glutaraldeído $(0,2-3,0 \%)$ têm sido utilizadas. A redução do tempo de isquemia entre a morte do animal e a retirada do tecido, a exposição e concentração do glutaraldeído, a duração da estocagem e o efeito residual dos aldeídos livres na redução da calcificação pós-implante são o foco das pesquisas clínicas e experimentais mais recentes. Algumas das novas tendências apontam para os processos de preservação sem glutaraldeído $(26,27)$.

- O excesso de tecido retido na parede aórtica do xenoenxerto parece determinar a extensão da futura calcificação. Embora esse fator possa ser modificado por agentes anti-cálcio; fatores intrínsecos do paciente como a baixa idade, constitui o determinante mais significativo da durabilidade tardia $(11,24)$. Os efeitos da calcificação da parede aórtica dos xenoenxertos na durabilidade dos folhetos permanece desconhecida, entretanto, a distensibilidade dinâmica normal e a extensibilidade da parede aórtica e a função do folheto podem ser perdidos substancialmente (25).

- As diversas técnicas de implante que variam desde a inserção subcoronária, cilindro intraluminal até a completa substituição da raiz da aorta, aumentam a complexidade operatória uma vez que pressupõem o implante de mais tecido da parede aórtica. Estudos multicêntricos validam as técnicas simples de implante como mais vantajosas e seguras ${ }^{(28)}$.

- O revestimento de partes dos xenoenxertos com Dacron é um fator importante que resulta na fixação fibrosa excessiva do tecido, na presença de panos ou de calcificação, tornando a bioprótese semi-rígida ("pseudo-suporte"). Esse efeito é minimizado com o implante supra-anular da valva CLOB, a qual possui pouco tecido aórtico e é destituída de revestimento com Dacron (29).
Características da Prótese Composta sem Suporte do Tipo Cryolife-O'Brien ${ }^{\text {TM }}$

A valva CryoLife-O'Brien modelo $300^{\mathrm{TM}}$ é um xenoenxerto composto construído a partir de três folhetos não-coronarianos obtidos de 3 valvas porcinas. Os folhetos são excisados cuidadosamente das valvas e fixados sob baixa pressão $(<2 \mathrm{mmHg})$ em glutaraldeído a $0,35 \%$. Cada folheto nãocoronariano é comparado quanto ao tamanho e simetria para assegurar a competência, evitar a abertura assincrônica, e promover máxima coaptação. Os três folhetos são então unidos através de uma sutura contínua das bordas livres da parede aórtica realizada na face posterior das comissuras utilizando 5-0 tevdek ${ }^{\mathrm{TM}}$. Não há revestimento externo com dacron, uma vez que a seleção de três folhetos não coronarianos exclui a barra muscular presente na base do folheto coronariano direito, característica natural das valvas porcinas.

A ausência de material adicional no anel valvar tem muitos benefícios dentre eles destacam-se: menor espessamento e enrijecimento anular, menor índice de reação do tipo corpo estranho e de infecção, mesmo nos pacientes com endocardite ativa.

Uma vez que a prótese CLOB possui pouco remanescente de tecido aórtico na base das cúspides; apenas uma linha de sutura se faz necessária para o seu implante, o que reduz significativamente os tempos de pinçamento aórtico e circulação extracorpórea (29).

\section{CASUÍSTICA E MÉTODOS}

\section{Técnica de Implante da Prótese Cryolife-O'Brien ${ }^{T M}$}

Antes da seleção do tamanho da valva e do início da sua lavagem, é importante realizar a medida intra-operatória direta da raiz da aorta, a qual pode ser auxiliada pelo ecocardiograma transesofágico realizado antes do pinçamento aórtico. Através de uma aortotomia transversa, a valva aórtica é excisada completamente, e 3 pontos eqüidistantes de polipropileno 3-0 são passados no ponto médio da porção supra-anular de cada seio aórtico, os quais orientam o implante simétrico. Em seguida, estes pontos são passados inferiormente de fora para dentro, envolvendo a maior extensão possível da prótese de modo a eliminar a margem de tecido protético subvalvar, e posteriormente amarrados, deixando os ramos dos fios do mesmo comprimento (Figura 2).

O implante prossegue com a sutura contínua do xenoenxerto iniciada no ponto médio de cada 


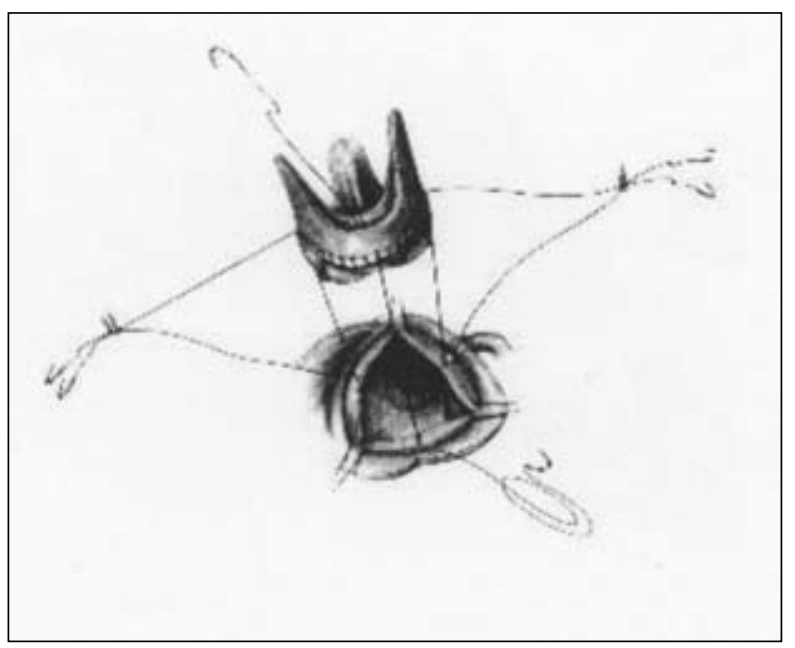

Fig. 2 - Três pontos eqüidistantes de polipropileno 3-0 na posição supra-anular próximo ao ponto médio de cada seio aórtico (reproduzida com permissão do Dr. Mark F. O'Brien).

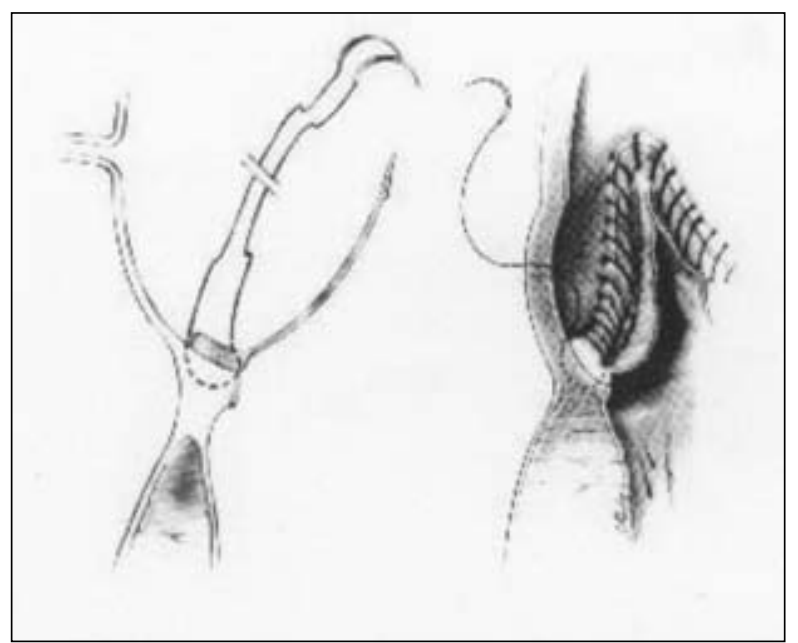

Fig. 3 - Sutura com pontos profundos e eqüidistantes no seio aórtico e na parede do xenoenxerto (reproduzida com permissão do Dr. Mark F. O'Brien).

Todos os pacientes foram acompanhados clínica e ecocardiograficamente no pós-operatório aos 6 dias (alta hospitalar), 6 meses, 12 meses e anualmente.

A análise estatística incluiu o teste "t-student", o teste exato de Fisher, e a análise atuarial de Kaplan-Meier para determinar a sobrevida, a liberdade de tromboembolismo, endocardite e reoperação por deterioração estrutural e por outras causas.

\section{RESULTADOS}

A mortalidade hospitalar (até 30 dias) foi $1,3 \% \quad 1 \%$ (4 pacientes) intervalo de confiança de $95 \%$. Os quatro óbitos não foram relacionados à troca valvar; e decorreram de: hemorragia retroperitonial secundária à inserção de balão intraaórtico; dissecção da aorta no orifício de canulação da cardioplegia (óbito ocorreu no 2 dia de pósoperatório); ruptura esplênica (queda da cama no 3 dia de pós-operatório) e morte súbita por arritmia no 29 dia de pós-operatório.

Ocorreram $28(9,2 \%)$ óbitos tardios, dos quais apenas $2(0,6 \%)$ foram relacionados à troca valvar. $\mathrm{Em}$ ambos os casos a etiologia era endocardite protética tardia tratada clinicamente. Todos os outros pacientes faleceram de causas naturais pertinentes à população senil; por exemplo câncer (7 casos), acidente vascular cerebral-AVC (6 casos), etc. A morbidade incluiu: AVC peri-operatório em $2(0,6 \%)$ pacientes, ambos tinham AVC prévio. Três $(0,9 \%)$ casos de tromboembolismo sistêmico 


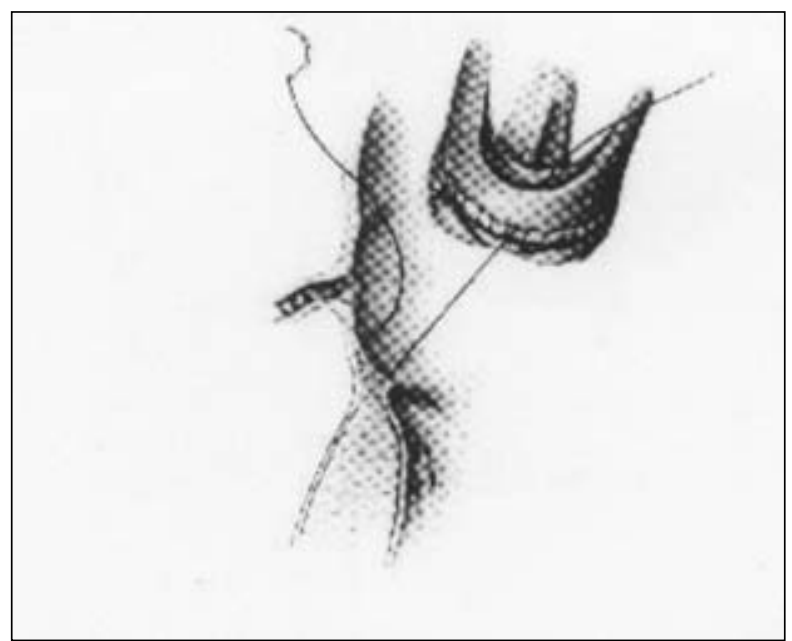

Fig. 4 - Linha de sutura única exteriorizada e amarrada no topo dos postes comissurais (reproduzida com permissão do Dr. Mark F. O'Brien).

precoce ocorreram em pacientes não anticoagulados que desenvolveram fibrilação atrial nos primeiros dias de pós-operatório. A anticoagulação passou a ser rotina a partir do octagésimo paciente, baseada na evidência de que a fibrilação atrial pós-operatória é complicação freqüente entre os pacientes idosos. Com a instituição da anticoagulação, não houve outros casos de tromboembolismo precoce.

Ocorreram $6(1,9 \%)$ casos de "leak" paravalvar, dos quais 4 foram apenas achados ecocardiográficos sem repercussão clínica. Nos outros 2 pacientes o "leak" foi decorrente de sutura frouxa em 1 e quebra da sutura no outro, necessitando a correção cirúrgica. Em ambos os casos a valva havia sido implantada utilizando-se fio de polipropileno 4-0. Nos últimos 240 pacientes utilizou-se fio de polipropileno 3-0, não sendo mais evidenciadas tais complicações.

Ocorreram $6(1,9 \%)$ casos de endocardite tardia, entre 1,5 e 5 anos de pós-operatório. Dois pacientes morreram sem operação. Três pacientes foram reoperados; dos quais 2 foram submetidos à substituição valvar, e no terceiro a prótese foi preservada. Um paciente foi curado clinicamente da endocardite sem sinais de degeneração valvar.

A incidência de reoperação foi $1,9 \%$ (6 casos). Houve $1(0,3 \%)$ caso de degeneração estrutural caracterizada por insuficiência valvar secundária à perfuração do folheto sem sinais de infecção, necessitando reoperação.

Os outros 5 casos de reoperação tiveram como indicação: endocardite ( 3 casos) e "leak" perivalvar ( 2 casos).
A análise atuarial de Kaplan-Meier para sobrevida e para liberdade de tromboembolismo, endocardite e, reoperação por degeneração estrutural e por outras causas está demonstrada na Tabela 1. Somente no 4 e 5 ano de seguimento é que se encontra um número suficiente de pacientes para atingir a significância estatística.

A avaliação clínica e ecocardiográfica seriada dos pacientes revelou excelentes resultados. Os gradientes transvalvar, a área do orifício efetivo, e o grau de incompetência de acordo como tamanho do anel em relação ao tamanho da valva implantada mostrou um gradiente médio de $7 \mathrm{mmHg}$ com progressão de estenose e insuficiência em apenas 1 $(0,3 \%)$ paciente.

Os 142 primeiros pacientes que receberam uma valva aórtica sem suporte do tipo CLOB foram comparados ecocardiograficamente com 40 pacientes submetidos à troca valvar aórtica no mesmo período utilizando uma valva porcina supra-anular com suporte do tipo CarpentierEdwards. O diâmetro pré-operatório da via de saída do ventrículo esquerdo (VE) era semelhante em ambos os grupos, $22 \mathrm{~mm} 0,4 \mathrm{~mm}$ e $23 \mathrm{~mm}$

$0,2 \mathrm{~mm}$ para a valva CLOB e Carpentier-Edwards, respectivamente $(p=N S)$. Apesar dos diâmetros semelhantes, foi possível implantar valvas maiores no grupo sem suporte $(26,3 \mathrm{~mm} \quad 2,4 \mathrm{~mm})$ do que no grupo com suporte $(23,7 \mathrm{~mm} 2,9 \mathrm{~mm})$, $\mathrm{p}<0,0001$.

Estudo ecocardiográfico aos 6 meses mostrou menor gradiente médio $(p \leq 0,0001)$ e maior orifício efetivo no grupo que recebeu a valva CLOB ( $p \leq 0,05$-Tabela 2).

Houve uma redução importante da massa ventricular esquerda em ambos os grupos aos 6 meses de pós-operatório, porém mais significativa no grupo da valva sem suporte $(p=0,05)$.

\section{COMENTÁRIOS}

Apesar dos 10 anos de seguimento de uma série pequena com a valva Toronto SPVTM, a durabilidade a longo prazo dos xenoenxertos sem suporte permanece desconhecida (20).

Esta revisão com a valva CLOB compreende um seguimento de 7 anos, no qual ocorreu apenas 1 caso de degeneração estrutural. Os resultados imediatos e a médio prazo são excelentes, embora o uso desta valva tenha sido predominante na população idosa; na qual a morte por diversas causas é fator de risco competitivo modificando a análise de ambas, durabilidade e sobrevida. 
TABELA 1

SOBREVIDA E LIBERDADE ATUARIAL DE EVENTOS MÓRBIDOS

\begin{tabular}{|c|c|c|c|c|}
\hline \multirow[b]{2}{*}{ Sobrevida ( $\geq 30$ dias) } & \multicolumn{2}{|c|}{4 ANOS } & \multicolumn{2}{|c|}{7 ANOS } \\
\hline & 86 & $3 \%$ & 68 & $7 \%$ \\
\hline \multicolumn{5}{|l|}{ Liberdade de: } \\
\hline Tromboembolismo & 93 & $2 \%$ & 93 & $2 \%$ \\
\hline Endocardite & 95 & $3 \%$ & 95 & $3 \%$ \\
\hline Reoperação - Degeneração tecidual & 99 & $1 \%$ & 99 & $1 \%$ \\
\hline Reoperação - Outras causas ("leak" perivalvar endocardite) & 96 & $2 \%$ & 96 & $2 \%$ \\
\hline
\end{tabular}

TABELA 2

DADOS COMPARATIVOS ENTRE A VALVA SEM SUPORTE CRYOLIFE-O'BRIEN ${ }^{\mathrm{TM}}(\mathrm{CLOB})$ E A VALVA SUPRA-ANULAR COM SUPORTE CARPENTIER-EDWARDS ${ }^{\mathrm{TM}}$

\begin{tabular}{lcc}
\hline & ALVA CLOB & VALVA CARPENTIER-EDWARDS \\
\hline NÚMERO DE PACIENTES & 142 & 40 \\
IDADE & 746 & 727 \\
SEXO (M/F) & $76 / 66$ & $25 / 15$ \\
HEMODINÂMICA (6 MESES PÓS-OPERATÓRIO) & & \\
GRADIENTE VALVAR MÉDIO $\left(\mathrm{mmHg}^{2}\right)$ & 84 & 145 \\
ÁREA DO ORIFÍCIO EFETIVO $\left(\mathrm{cm}^{2}\right)$ & $2,30,4$ & $2,10,7$ \\
MASSA VENTRICULAR ESQUERDA & $27264 \mathrm{~g}$ & $25758 \mathrm{~g}$ \\
PRÉ-OPERATÓRIA & $22072 \mathrm{~g}$ & $23074 \mathrm{~g}$ \\
6 MESES PÓS-OPERATÓRIO & $\mathrm{p}=0,0001$ & $\mathrm{p}=0,02$ \\
VALOR DE p PRÉ E PÓS-OPERATÓRIO & & $\mathrm{p}=0,05$ \\
\hline COMPARAÇÃO DO VALOR DE p PÓS-OPERATÓRIO & & \\
\hline CLOB= valva CryOLife-O'Brien; & & \\
\hline
\end{tabular}

As próteses sem suporte têm alguns dos atributos dos homoenxertos aórticos. Talvez a qualidade mais importante, quando comparadas às próteses com suporte, seja a hemodinâmica favorável principalmente nas raízes da aorta pequena ${ }^{(28)}$. O orifício efetivo; uma vez sentada na raiz da aorta, é maior quando comparado às outras biopróteses e próteses mecânicas ${ }^{(18,31)}$. Isto também se aplica à prótese CLOB, na qual, por exemplo, em um paciente com anel valvar de $25 \mathrm{~mm}$ pode ser implantada uma prótese $27 \mathrm{~mm}$ na posição supra-anular.

Embora os folhetos dos xenoenxertos sejam um pouco rígidos, eles produzem um baixo gradiente transvalvar quase tão bom quanto os homoenxertos. Em estudo anterior já havia sido demonstrado que as valvas sem suporte promovem uma regressão ventricular mais rápida e mais completa do que as com suporte (18). JIN et al. (21) compararam os dados hemodinâmicos dos homoenxertos, prótese sem suporte Toronto SPVTM e próteses com suporte. Ambos os tipos sem suporte (homoenxerto e Toronto $\mathrm{SPV}^{\mathrm{TM}}$ ) causaram menor resistência à ejeção ventricular esquerda e produziram melhora mais precoce da função ventricular esquerda $e$, consequentemente, uma resolução mais completa da hipertrofia ventricular. Esses achados importantes também foram verificados por DEL RIZZO et al. (31) com a valva Toronto SPVTM.

A vantagem teórica e provavelmente o interesse clínico no uso das próteses sem suporte é a alta 
durabilidade devido à melhor distribuição das forças físicas durante os movimentos dos folhetos. Esta dissipação de forças não ocorre nas valvas com suporte ${ }^{(24-26)}$.

Há também alguma evidência experimental que as valvas aórticas sem suporte são menos susceptíveis à calcificação (27). Entretanto, serão necessários outros 5 anos de seguimento para que a melhor durabilidade das valvas sem suporte atinja significância estatística.

Alguns aspectos devem ser observados antes do implante de uma valva sem suporte:

- A calcificação excessiva da raiz da aorta pode dificultar a sutura do tecido aórtico supra-anular e peri-comissural. Isto também se aplica aos modelos que requerem implante subcoronário. Nestes casos uma bioprótese com suporte (ex: valva de pericárdio) parece ser a melhor alternativa.

- $O$ anel aórtico grande $(\geq 30 \mathrm{~mm})$ pode constituir uma dificuldade técnica, sendo necessária a plicatura do anel (32); ou mesmo uma contraindicação para o uso se a prótese de escolha for a CLOB, uma vez o maior tamanho disponível é $29 \mathrm{~mm}$.

- A assimetria acentuada da raiz da aorta, embora rara em idosos, pode produzir resultados menos favoráveis com o implante subcoronário de uma valva sem suporte do que aquele obtido com uma valva de pericárdio com suporte. A alternativa para estes casos poderia ser a substituição da raiz da aorta por um xenoenxerto sem suporte (30).

Um outro aspecto importante na indicação das valvas sem suporte é a faixa etária-alvo. Sabe-se da literatura(33) que os resultados com os xenoenxertos com suporte são pobres nas crianças e adultos jovens até a idade de 50 a 60 anos, sendo portanto, desaconselhável o uso liberal das próteses biológicas na população jovem, a menos que os homoenxertos não sejam disponíveis ou que haja contra-indicação formal para o uso dos anticoagulantes. Entretanto, as próteses sem suporte poderiam ser uma altenativa neste grupo jovem de pacientes dada à maior durabilidade potencial.
As próteses com e sem suporte produzem resultados clínicos imediatos semelhantes; entretanto, a médio prazo as próteses sem suporte apresentam melhor rendimento hemodinâmico caracterizado por uma maior área de orifício efetivo, menor gradiente médio e maior regressão ventricular esquerda $(18,21)$.

No "The Prince Charles Hospital" a experiência de 7 anos com a valva CLOB tem sido satisfatória com apenas $4(1,3 \%)$ óbitos numa série de 307 pacientes consecutivos. O implante rápido, permitindo a realização de procedimentos associados em $56 \%$ dos casos constituiu uma vantagem importante. A simetria da raiz da aorta parece ter sido um outro fator contributivo para os bons resultados com esse tipo de valva, uma vez que a maioria dos pacientes que receberam esta valva possuiam uma valva aórtica nativa tricúspide estenosada por calcificação degenerativa adquirida. Tal simetria, dado o reduzido remanescente de tecido aórtico presente nestas valvas, favorece o implante supra-anular utilizando-se linha de sutura contínua única (33).

\section{CONCLUSÕES}

- A baixa mortalidade, o bom rendimento hemodinâmico caracterizado por baixos gradientes, bom orifício efetivo, regressão ventricular esquerda significativa e poucas complicações relacionadas ao implante valvar, garantem a segurança e eficácia das próteses aórticas sem suporte;

- A experiência com a valva CLOB na população idosa demonstrou resultados excelentes a curto e médio prazos, entretanto, a durabilidade a longo prazo permanece desconhecida;

- O conhecimento da durabilidade das próteses sem suporte permitirá estender o seu uso à população mais jovem;

Vigilância rigorosa será necessária para determinar a durabilidade das valvas sem suporte aos 10-12 anos; período em que as valvas porcinas com suporte começam a demonstrar deterioração estrutural. 
Nina V J S \& O'Brien M F - Stentless aortic valve: what we have learnt. Rev Bras Cir Cardiovasc $2001 ; 16(2): 119-27$.

ABSTRACT: Objective: The aim of this study is to debate the current status and justification for continued use of stentless valves, outlining the clinical results with the CryoLife-O'Brien composite porcine stentless valve ${ }^{\mathrm{TM}}$ (CLOB).

Material and Methods: Between December 1992 and February 2000, 307 patients underwent aortic valve replacement with a CLOB valve. The mean age was 73 years (range $59-89$ years with $16 \%$ being 80 years and over). All patients were assessed clinically and by echocardiography postoperatively at 6 days, 6 months, 12 months and yearly thereafter.

Results: The hospital mortality was $1.3 \%$ (4 valve-unrelated cases). Late mortality occurred with 28 patients $(9.2 \%)$, of which only 2 were valve-related (late endocarditis). Morbid events have included $2(0.6 \%)$ perioperative strokes, $2(0.6 \%)$ perivalvar leaks, $6(1.9 \%)$ endocarditis and $1(0.3 \%)$ structural deterioration. Reoperation was required in 6 patients (1.9\%): 3 for endocarditis, 2 for perivalvar leak and 1 for structural deterioration. Serial echocardiography demonstrated a mean gradient of $7 \mathrm{mmHg}$ with a very low incidence of trivial incompetence on Doppler, and significant left ventricular hypertrophic regression $(p=0.05)$.

Conclusion: Stentless valves have produced excellent early and mid-term results. However, surveillance is required to determine the durability at 10 to 12 years, a time when stented porcine valves begin to show failure from structural deterioration.

DESCRIPTORS: Heart valve prosthesis. Heart valve prosthesis implantation, methods.

\section{REFERÊNCIAS BIBLIOGRÁFICAS}

1 Binet J P, Duran C G, Carpentier A, Langlois J Heterologous aortic valve transplantation. Lancet 1965; 2: 1275-6.

2 O'Brien M F - Heterograft aortic valves for human use: valve bank, techniques of measurement and implantation. J Thorac Cardiovasc Surg 1967; 53: 392-7.

3 Carpentier A, Lemaigre G, Robert L, Carpentier S, Dubost C - Biological factors affecting long-term results of valvular heterografts. J Thorac Cardiovasc Surg 1969; 58: 467-83.

4 O'Brien M F, Neilson G H, Galea E G et al. - Heterograft valves: an analysis of clinical results of valve replacement. Circulation 1970; 41(5 Suppl II): II16-9.

5 Carpentier A, Deloche A, Relland J et al. - Six year follow-up of glutaraldehyde-preserved heterografts: with particular reference to the treatment of congenital valve malformations. J Thorac Cardiovasc Surg 1974; 68: $771-82$

6 Jamieson W R, Burr L H, Munro A I, Miyagishima R T, Gerein A N - Cardiac valve replacement in the elderly: clinical performance of biological prostheses. Ann Thorac Surg 1989; 48: 173-85.

7 Jamieson W R - Modern cardiac valve devices: bioprostheses and mechanical prostheses: state of the art. J Card Surg 1993; 8: 89-98.
8 Burdon T A, Miller D C, Oyer P E et al. - Durability of porcine valves at fifteen years in a representative North American patient population. J Thorac Cardiovasc Surg 1992; 103: 228-52.

9 Magilligan Jr. D J, Lewis Jr. J W, Tilley B, Peterson $\mathrm{E}$ - The porcine bioprosthetic valve: twelve years later. J Thorac Cardiovasc Surg 1985; 89: 499-507.

10 Williams D B, Danielson G K, McGoon D C, Puga F J, Mair D D, Edwards W D - Porcine heterograft valve replacement in children. $J$ Thorac Cardiovasc Surg 1982; 84: 446-50.

11 Dunn J M - Porcine valve durability in children. Ann Thorac Surg 1981; 32: 357-68.

12 Hammond G L, Geha A S, Kopf G S, Hashim S W Biological versus mechanical valves: analysis of 1.116 valves inserted in 1.012 adult patients with a 4.818 patient-year and a 5.327 valve-year follow-up. $J$ Thorac Cardiovasc Surg 1987; 93: 182-98.

13 Thubrikar M J, Deck J D, Aouad J, Nolan S P - Role of mechanical stress in calcification of aortic bioprosthetic valves. J Thorac Cardiovasc Surg 1983; 86: 115-25.

14 Pupello D F, Bessone L N, Hiro S P, Lopez-Cuenca E, Glatterer Jr. M S, Ebra G - The CarpentierEdwards bioprosthesis: a comparative study analysing failure rates by ages. J Card Surg 1988; 3(3 Suppl): 369-74. 
15 Ferrans $\mathrm{V} J$, Boyce S W, Billingham M E, Jones $M$, Ishihara T, Roberts W C - Calcific deposits in porcine bioprostheses: structure and pathogenesis. $A m ~ J$ Cardiol 1980; 46: 721-34.

Jamieson W R - Stented porcine bioprosthesis-durability and outcomes, 1975-1993. In: Piwnica A, Westaby S, eds. Stentless bioprosthesis. Oxford: Isis Medical Media, 1995: 24-35.

17 Angell W W, Pupello D F, Bessone L N, Hiro S P, Brock $\mathrm{J} C$ - Effect of stent mounting on tissue valves for aortic valve replacement. J Card Surg 1991; 6 (Suppl 4): 595-9.

18 Thomson $\mathrm{H}$ L, O'Brien M F, Almeida A A, Tesar P J, Davison M B, Burstow D J - Haemodynamics and left ventricular mass regression. a comparison of the stentless, stented and mechanical aortic valve replacement. Eur J Cardiothorac Surg 1998; 13: 572-5.

David T E, Puschmann R, Ivanov J et al.- Aortic valve replacement with stentless and stented porcine valves: a case-match study. J Thorac Cardiovasc Surg 1998; 116: $236-41$.

20 David T E, Feindel C M, Scully H E, Bos J, Rakowski $\mathrm{H}$ - Aortic valve replacement with stentless porcine aortic valves: a ten year experience. J Heart Valve Dis 1998; 7: 250-4.

21 Jin X Y, Zhang Z M, Gibson D G, Yacoub M H, Pepper $J R$ - Effect of valve substitute on changes in left ventricular function and hypertrophy after aortic valve replacement. Ann Thorac Surg 1996; 62: 683-90.

O'Brien M F - The CryoLife-O'Brien composite aortic stentless xenograft: surgical technique of implantation. Ann Thorac Surg 1995; 60 (2 suppl): S410-3.

Christie G W \& Barratt-Boyes B G - On stress reduction in bioprosthetic heart valve leaflets by the use of a flexible stent. J Card Surg 1991; 6: 476-81.

24 Sabbah H N, Hamid M S, Stein P D - Mechanical stresses on closed cusps of porcine bioprosthetic valves: correlation with sites of calcification. Ann Thorac Surg 1986; 42: 93-6.

Thubrikar M J, Deck J D, Aouad J, Nolan S P - Role of mechanical stress in calcification of aortic bioprosthetic valves. J Thorac Cardiovasc Surg 1983; 86: 115-25.

O'Brien M F, Goldstein S, Walsh S, Black K S, Elkins $R$, Clarke D - The Synergraft valve: a new acellular (nonglutaraldehyde-fixed) tissue heart valve for autologous recellularization. Semin Thorac Cardiovasc Surg 1999; 11 (4 Suppl I):194-200.

Hazekamp M G, Goffin Y A, Huysmans H A - The value of stentless biovalve prosthesis. An experimental study. Eur J Cardiothorac Surg 1993; 7: 514-9.

Hvass U, Palatianos G M, Frassani R, Puricelli C, O'Brien $M$ - Multicenter study of stentless valve replacement in small aortic root. J Thorac Cardiovasc Surg 1999; 117: $267-72$.

O'Brien M F, Gardner M A H, Garlick R B et al. - The CryoLife-O'Brien stentless aortic porcine xenograft valve. J Card Surg 1998; 13: 376-85.

O'Brien M F - Implantation technique of the CryoLifeO'Brien stentless xenograft aortic valve: the simple, rapid, and correct way to implant and the errors to avoid. Semin Thorac Cardiovasc Surg 1999; 11(4 Suppl 1): 121-5.

31 Del Rizzo D F, Goldman B S, Christakis G T, David $T E$ - Hemodynamic benefits of the Toronto stentless valve. J Thorac Cardiovasc Surg 1996; 112: $1431-46$.

32 Petracek M R, Shuman T A, Pirolo J S, Tedder M, Ball S K, Graves D - Use of Toronto stentless porcine valve in patients with aortic dilation. Semin Thorac Cardiovasc Surg 1999; 11(4 Suppl 1): 74-8.

33 McGiffin D C, Galbraith A J, O'Brien M F et al. - An analysis of valve re-replacement after aortic valve replacement with biologic devices. J Thorac Cardiovasc Surg 1997; 113: 311-8. 